\title{
Preoperative prognostic factors for severe diffuse secondary peritonitis : a retrospective study
}

\section{Tolonen, Matti}

2016-08

Tolonen , M , Sallinen , V , Mentula , P \& Leppaniemi , A 2016 , ' Preoperative prognostic factors for severe diffuse secondary peritonitis : a retrospective study ' , Langenbecks Archives of Surgery , vol. 401 , no. 5 , pp. 611-617 . https://doi.org/10.1007/s00423-016-1454-8

http://hdl.handle.net/10138/224158

https://doi.org/10.1007/s00423-016-1454-8

publishedVersion

Downloaded from Helda, University of Helsinki institutional repository.

This is an electronic reprint of the original article.

This reprint may differ from the original in pagination and typographic detail.

Please cite the original version. 


\title{
Preoperative prognostic factors for severe diffuse secondary peritonitis: a retrospective study
}

\author{
Matti Tolonen $^{1} \cdot$ Ville Sallinen $^{1,2} \cdot$ Panu Mentula $^{1} \cdot$ Ari Leppäniemi $^{1}$
}

Received: 18 March 2016/Accepted: 20 May 2016/Published online: 30 May 2016

(C) Springer-Verlag Berlin Heidelberg 2016

\begin{abstract}
Purpose The aim of this study was to analyse preoperative risk factors for mortality or intensive care unit admission to describe severe peritonitis.

Methods This was a single academic centre retrospective study of consecutive adult patients operated for diffuse secondary peritonitis between 2012 and 2013. Patients with appendicitis or cholecystitis were excluded. Independent risk factors were identified using binary and ordinal logistic regression.

Results A total of 223 patients were analysed. Overall 30-day mortality was $14.5 \%$. Postoperatively, $32.3 \%$ of patients were admitted into the intensive care unit (ICU). Independent risk factors for severe peritonitis were septic shock (odds ratio (OR) 37.94, $95 \%$ confidence interval (CI) 14.52-99.13), chronic kidney insufficiency (OR 5.98 (95\% CI 1.56-22.86), severe sepsis (OR 4.80, $95 \%$ CI 2.10-10.65) and cardiovascular disease (OR 2.58, $95 \%$ CI 1.22-5.47). Patients lacking these factors had no mortality. ICU admission was refused in $24(10.8 \%)$ patients with $70.8 \%$ mortality. In a
\end{abstract}

This study was presented at National Operative Days Conference, 25 November 2015, Helsinki, Finland

Electronic supplementary material The online version of this article (doi:10.1007/s00423-016-1454-8) contains supplementary material, which is available to authorized users.

Matti Tolonen

matti.tolonen@hus.fi

1 Department of Abdominal Surgery, University of Helsinki and Helsinki University Hospital Meilahti, Haartmaninkatu 4, 00290 Helsinki, Finland

2 Department of Transplantation and Liver Surgery, University of Helsinki and Helsinki University Hospital, Helsinki, Finland subgroup of patients without treatment limitations $(n=190)$, independent risk factors for weighted outcome of ICU admission or mortality were septic shock (OR 11.89, $95 \%$ CI 4.98 28.40), severe sepsis (OR 5.56, $95 \%$ CI 2.39-12.89), metastatic malignant disease or lymphoma (OR 3.11, $95 \%$ CI 1.34-7.20) and corticosteroid use (OR 2.98, $95 \%$ CI 1.187.51). When receiving full level of care, patients with preoperative organ dysfunctions in this subgroup had $8.2 \% 30$-day mortality.

Conclusions Preoperative organ dysfunctions, chronic kidney insufficiency and cardiovascular disease are the most important risk factors for severe peritonitis. Without these risk factors, patients had no mortality.

Keywords Laparotomy · Intraabdominal infections · Sepsis · Critical care $\cdot$ Multiple organ failure

\section{Introduction}

Perforation of gastrointestinal (GI) tract leading to diffuse secondary peritonitis is a serious condition with substantial mortality (20-40\%) and morbidity [1-3]. Peritonitis is classified as diffuse (or generalised) if infection spreads widely into abdominal cavity as opposed to local peritonitis in which infection is limited into a part of abdominal cavity [1]. Diffuse peritonitis forms about $40-45 \%$ of all operatively managed abdominal infections [4]. The most severe cases, associated with persisting organ dysfunctions, are treated postoperatively in intensive care unit (ICU) $[1,5]$. These severe cases constitute about $30-40 \%$ of diffuse peritonitis with mortality up to $70 \%$ $[1,2,4]$. Elective gastrointestinal surgical operations are also associated with a significant risk (5-10\%) of developing postoperative peritonitis due to anastomotic dehiscence [1], with associated mortality even higher compared to secondary 
peritonitis [6]. Abdominal infections are the second most common source for ICU-treated sepsis, following pulmonary infections [3]. The classic principles in treating secondary peritonitis include intravenous fluid resuscitation, support of organ function, broad-spectrum systemic antimicrobial medication, timely surgical source control, restoration of GI function and peritoneal lavage with evacuation of infectious material $[7,8]$. It is of great importance to identify patients at high risk of severe peritonitis as early as possible since delays in antibiotic treatment, fluid resuscitation and surgical source control have been shown to increase mortality, even though the correct timing of surgical source control is still controversial [8-11].

There are a number of studies and reviews that have analysed and listed prognostic factors in peritonitis $[1,2,4$, $6,12-15]$. None of these previous studies specifically concentrate on preoperative variables. However, decisions of triage for the operating room need to be done already in the emergency department based on the available information. Also, surgical decision making in the first operation has to be done based on this information combined with the degree of anatomical derangement. In a review article by Pieracci and Barie, factors associated with mortality in secondary peritonitis were shock, increasing age, increasing Acute Physiology and Chronic Health Evaluation (APACHE) II score, isolation of enterococci, impaired consciousness, inadequate empiric antibiotics, poor nutritional status, cardiovascular disease, inability to obtain source control, immunosuppression, hypoalbuminemia, thrombocytopenia, diffuse versus localised peritonitis, over 24-h delay before source definitive intervention, subsequent nosocomial infection and protein $\mathrm{C}$ concentration less than $66 \%$ of normal [1].

The aim of this study was to analyse patients with diffuse secondary peritonitis and identify association of readily available preoperative risk factors to severe peritonitis, which was described as 30-day mortality or ICU admission. Comprehending these risk factors would help in identifying patients in the biggest risk for severe peritonitis and in need of timely actions and new treatment strategies.

\section{Material and methods}

This study was conducted as a retrospective cohort study from a single academic centre that serves both as a secondary and a tertiary referral hospital. Institutional human research review committee approved the study design. This study was an observational retrospective cohort study, and neither informed consent nor ethics committee's approval was needed. Electronic operating room log was browsed for all abdominal emergency surgery cases, which were further analysed from electronic patient records, and all consecutive operatively treated adult patients with diffuse secondary peritonitis due to perforation in GI tract between January 1, 2012 and
December 31, 2013 were included in the study cohort. Patients with appendicitis or cholecystitis were excluded due to their better prognosis and straightforward treatment protocols $[2,13,16]$. Further, four patients were excluded from the study group because they were already receiving ICU care due to another disease (two patients with severe pancreatitis, one early postoperative period after lung transplantation and one postoperative period after operation for ruptured abdominal aortic aneurysm) during the time of GI perforation. Additionally, one patient was excluded since first operation for peritonitis was done in another hospital. A total of 223 patients were included in the final study cohort.

Missing values are stated in the tables, and they were either considered to be within reference values or discarded from analysis depending on clinical rationale.

\section{Definitions}

Comorbidities were classified according to Charlson Comorbidity Index (CCI) [17]. Other calculated scores included APACHE II, Mannheim Peritonitis Index (MPI) and the American Society of Anesthesiologists (ASA) physical status classification $[18,19]$. Sepsis was classified according to previously reported diagnostic criteria for sepsis, severe sepsis (sepsis with organ dysfunction) and septic shock [5].

Signs of preoperative organ dysfunction were defined according to Sequential Organ Failure Assessment (SOFA) score of one point or more in each organ system when sufficient data was available [20]. Due to the retrospective nature of the data and emergency setting, it was not possible to define exact SOFA scores. Instead, an extended criterion for organ dysfunction was used in order to detect early organ dysfunction. When respiratory $\mathrm{PaO} 2 / \mathrm{FiO} 2$ was not measured, pulse oximetry saturation less than $90 \%$ or respiratory rate constantly over 25 per minute was used. Because daily urine output was not measurable in any of the acute patients, over $50 \%$ rise in baseline plasma creatinine or hourly urine output less than $0.5 \mathrm{ml} / \mathrm{kg}$ under fluid resuscitation was used instead. When mean arterial pressure (MAP) was not available in patients who did not have an arterial line, the following equation $[(2 \times$ diastolic bp $)+$ systolic bp $] / 3$ to estimate MAP was used.

Main outcome was a composite outcome of 90-day mortality or ICU admission.

\section{Statistical analyses}

Patient characteristics and perioperative data are presented in number and percentage of patients, mean and median, range and interquartile range (IQR) where appropriate. Statistical analyses were performed using Fischer's exact test, chisquared test, $t$ test, logistic regression analysis with forward stepwise selection and ordinal regression analysis where appropriate. For continuous variables, Shapiro-Wilk's test was 
used to define normality of distribution. Odds ratio (OR) values are presented with $95 \%$ confidence interval $(\mathrm{CI})$, and two-tailed $p$ value below 0.05 was considered significant. For logistic and ordinal regression analyses, robust and easily measurable variables were chosen, which could be used in clinical work. From univariate analysis, variables with $p<0.20$ were chosen, however avoiding multicollinearity between variables. Cardiovascular diseases, other than arterial hypertension, were combined as a single risk factor. In binary logistic regression analysis, all variables were categorical, goodness of fit was tested using Hosmer-Lemeshow test and model performance was tested using Nagelkerke $R^{2}$. In ordinal logistic regression analysis, Pearson's chi-squared goodness-of-fit test and Nagelkerke $R^{2}$ were used. Cutoff points for factors in analyses were chosen according to definitions in previous studies, clinical sense and with the help of receiver operating characteristic (ROC) curves.

All patients' data were analysed more than 90 days postoperatively. Analyses were performed using SPSSC Statistics version 22 for Mac (IBMC, Armonk, NY, USA).

\section{Results}

Two-hundred and twenty-three patients with diffuse peritonitis were analysed. Patient characteristics (Table 1) and intraoperative and postoperative data (Table 2) are presented in distinct tables to describe the study cohort, diagnoses, severity of comorbidities, preoperative physiology, intraoperative variables and postoperative outcome. Shortly, patients had a mean age of 63.3 years; $53.4 \%$ were male, $93.6 \%$ lived at home and $48.0 \%$ were ASA class 4 (Table 1). Further, median MPI was $28 ; 25.6 \%$ had a postoperative peritonitis, $54.7 \%$ had perforation of the colon and $74.4 \%$ had a purulent peritonitis (Table 2).

Overall 30-day mortality was $14.5 \%(n=33)$ and 90 -day mortality was $22.0 \%(n=49)$. Postoperatively, $72(32.3 \%)$ patients were admitted to the ICU with a 30-day mortality rate of $16.7 \%(n=12)$. The 30 -day mortality rate for all non-ICUtreated patients was $13.9 \%(n=21)$.

Preoperative factors associated with 30-day mortality or ICU admission in univariate analysis are presented in Supplementary Table 1. Several preexisting conditions, medications, emergency department findings and organ dysfunctions were significantly associated with ICU admission or mortality. If patients had signs of severe sepsis $(n=46$, $20.6 \%)$ or septic shock $(n=58,26.0 \%)$ at admission, 30 day mortality rates were 21.7 and $29.3 \%$ and ICU admittance rates were 34.8 and $67.2 \%$, respectively.

In a multivariate logistic regression analysis, the severity of sepsis, chronic kidney insufficiency and preexisting cardiovascular disease were independent predictors of 30day mortality or ICU admission (Table 3). Area under receiver operating characteristic (AUROC) for this multivariate model
Table 1 Patient characteristics

\begin{tabular}{|c|c|}
\hline \multicolumn{2}{|l|}{ Baseline } \\
\hline Total number of patients & 223 \\
\hline Sex, male/female, $n(\%)$ & $119 / 104(53.4 / 46.6)$ \\
\hline Age, years, mean (range) & $63.3(17-94)$ \\
\hline BMI, kg/m², mean (range) & $25.3(13-51)^{\mathrm{a}}$ \\
\hline Prehospital living conditions, $n(\%)$ & $\mathrm{a}$ \\
\hline Home & $208(93.6)$ \\
\hline Institutionalised & $13(5.9)$ \\
\hline \multicolumn{2}{|l|}{ Medications, $n(\%)$} \\
\hline Immunosuppressives (excluding corticosteroids) & $17(7.6)$ \\
\hline Corticosteroids & $41(18.4)$ \\
\hline Chemotherapy & $32(14.3)$ \\
\hline Anticoagulants (chronic use) & $36(15.7)$ \\
\hline Antithrombotics & $41(18.4)$ \\
\hline \multicolumn{2}{|l|}{ Comorbidities, $n(\%)$} \\
\hline Myocardial infarction & $16(7.2)$ \\
\hline Congestive heart failure & $26(11.7)$ \\
\hline Arteriosclerosis & $19(8.5)$ \\
\hline Cerebrovascular disease (except haemiplegia) & $26(11.7)$ \\
\hline Dementia & $25(11.2)$ \\
\hline COPD & $14(6.3)$ \\
\hline Connective tissue disease & $18(8.1)$ \\
\hline Ulcer disease & $57(25.6)$ \\
\hline Diabetes & $38(17.0)$ \\
\hline Renal insufficiency (moderate or severe) & $18(8.1)$ \\
\hline Haemiplegia & $3(1.3)$ \\
\hline Malignancy & $72(32.3)$ \\
\hline Malignant lymphoma & $9(3.9)$ \\
\hline \multicolumn{2}{|l|}{ Solid malignant tumour } \\
\hline Without metastasis & $26(11.7)$ \\
\hline With metastasis & $34(15.2)$ \\
\hline Liver disease (moderate or severe) & $3(1.3)$ \\
\hline AIDS & $1(0.4)$ \\
\hline Coronary artery disease without infarction & $31(13.9)$ \\
\hline Previous thromboembolic disease & $21(9.4)$ \\
\hline Arterial hypertension & $109(48.9)$ \\
\hline \multicolumn{2}{|l|}{ Charlson Comorbidity Index (CCI) } \\
\hline Mean (median) & $2.6(2)$ \\
\hline Range (IQR) & $0-15(1-4)$ \\
\hline \multicolumn{2}{|l|}{ ASA class } \\
\hline 1 & $22(9.9)$ \\
\hline 2 & $27(12.1)$ \\
\hline 3 & $67(30.0)$ \\
\hline 4 or more & $107(48.0)$ \\
\hline
\end{tabular}

BMI $n=19$, prehospital living conditions $n=2$

$B M I$ body mass index, COPD chronic obstructive pulmonary disease, $A I D S$ acquired immune deficiency syndrome, IQR interquartile range, ASA American Society of Anesthesiologist

${ }^{\mathrm{a}}$ Missing values (discarded from analysis) 
Table 2 Intraoperative and postoperative data

\begin{tabular}{|c|c|}
\hline \multicolumn{2}{|l|}{ Intraoperative period, $n(\%)$} \\
\hline \multicolumn{2}{|l|}{ Reason for perforation } \\
\hline Disease & $164(74.0)$ \\
\hline Postoperative & $57(25.6)$ \\
\hline Not known & $1(0.4)$ \\
\hline \multicolumn{2}{|l|}{ Site of perforation and related diagnosis } \\
\hline Upper GI tract (stomach and duodenum) & $61(27.4)$ \\
\hline Peptic ulcer perforation & $52(23.3)$ \\
\hline Tumour perforation & $3(1.3)$ \\
\hline Ananstomosis dehiscence & $2(0.9)$ \\
\hline Iatrogenic & $2(0.9)$ \\
\hline Other & $2(0.9)$ \\
\hline Small bowel & $40(17.9)$ \\
\hline Iatrogenic & $12(5.4)$ \\
\hline Anastomosis dehiscence & $7(3.1)$ \\
\hline Diverticulitis & $5(2.2)$ \\
\hline Strangulation & $5(2.2)$ \\
\hline Crohn's disease & $3(1.3)$ \\
\hline Tumour perforation & $3(1.3)$ \\
\hline Other & $5(2.2)$ \\
\hline Colon & $122(54.7)$ \\
\hline Diverticulitis & $44(19.7)$ \\
\hline Anastomosis dehiscence & $28(12.6)$ \\
\hline Tumour & $14(6.3)$ \\
\hline Iatrogenic & $6(2.7)$ \\
\hline Stercoral & $6(2.7)$ \\
\hline Benign stricture & $5(2.2)$ \\
\hline Pseudo-obstruction & $4(1.8)$ \\
\hline Volvulus & $4(1.8)$ \\
\hline Other & $11(4.9)$ \\
\hline \multicolumn{2}{|l|}{ Exudate } \\
\hline Clear & $5(2.2)$ \\
\hline Purulent & $166(74.4)$ \\
\hline Faecal & $52(23.3)$ \\
\hline Source control achieved & $216(96.9)$ \\
\hline \multicolumn{2}{|l|}{ Open abdomen } \\
\hline At primary operation & $10(4.5)$ \\
\hline At subsequent operations & $7(3.1)$ \\
\hline \multicolumn{2}{|l|}{ Duration of operation, minutes } \\
\hline Mean (median) & $117(107)$ \\
\hline Range (IQR) & $30-428(75-144)$ \\
\hline Manneheim Peritonitis Index (MPI) & a \\
\hline Mean (median) & $27.7(28)$ \\
\hline Range (IQR) & $12-43(23-33)$ \\
\hline \multicolumn{2}{|l|}{ Postoperative period, $n(\%)$} \\
\hline ICU admittance & $72(32.3)$ \\
\hline \multicolumn{2}{|l|}{ Length of ICU stay, days } \\
\hline Mean (median) & $7.2(4.5)$ \\
\hline Range (IQR) & $1-39(3-8)$ \\
\hline \multicolumn{2}{|l|}{ Highest SOFA score in ICU } \\
\hline Mean (median) & $7.9(8)$ \\
\hline Range (IQR) & $2-17(5-10)$ \\
\hline 30/90-day mortality in ICU-admitted patients & $12 / 19(16.7 / 26.4)$ \\
\hline Reoperations & \\
\hline Planned/unplanned & $12 / 44(5.4 / 19.7)$ \\
\hline \multicolumn{2}{|c|}{ Limitation of treatments (DNR, no ICU or no renal replacement therapy) } \\
\hline Immediately postoperatively & $24(10.8)$ \\
\hline Later in treatment & $21(9.4)$ \\
\hline \multicolumn{2}{|l|}{ Mortality, $n(\%)$} \\
\hline Mortality 30 days & $33(14.8)$ \\
\hline Mortality 90 days & $49(22.0)$ \\
\hline
\end{tabular}

GI gastrointestinal, $I Q R$ interquartile range, $I C U$ intensive care unit, SOFA Sequential Organ Failure Assessment, DNR do not resuscitate

${ }^{\text {a }}$ Missing values (discarded from analysis), $n=6$ was 0.87 (CI 0.82-0.92, $p<0.001$ ), Hosmer-Lemeshow goodness-of-fit chi-squared test was 11.93 and degrees of freedom was 4 with significance 0.180 , showing that the model has adequate fit. Model performance was tested using Nagelkerke $R^{2}$ with a result of 0.49 .

When analysing patients with none of the independent risk factors $(n=86,38.6 \%)$, there was no 30 -day mortality and ICU admittance rate was $7.0 \%(n=6)$. If patients had no signs of organ dysfunction preoperatively $(n=121,54.3 \%)$, the rates were $5.0 \%(n=6)$ and $14.0 \%(n=17)$, respectively.

Twenty-four $(10.8 \%)$ patients were deemed too sick to benefit from ICU treatment immediately postoperatively and were refused ICU admission. This group had $70.8 \%(n=17)$ 30-day mortality and $87.5 \%(n=21)$ 90-day mortality. Characteristics of this patient group are summarised in Table 4. These patients had a median age of 74 years; $20.8 \%$ were institutionalised, $41.7 \%$ had a metastatic malignant disease or lymphoma and $83.4 \%$ had signs of organ dysfunction preoperatively. In the ICU, a decision to withdraw life-sustaining treatment was done in 21 (9.4\%) patients, who did not respond to care.

Patients who did not need ICU level care and limitation of treatments were not made $(n=106,47.5 \%)$ had no 30 -day mortality.

\section{Subgroup analysis}

An analysis of patients without limitations of treatment during the first 3 days of hospitalisation ( $n=190$; Table 5) was conducted. This was done in order to separately analyse patients who received full level of care with emphasis on mortality over ICU admittance as outcome. In this group, 30-day mortality was $4.7 \%(n=9)$ and $32.6 \%(n=62)$ of patients were admitted into the ICU with $8.1 \%(n=5)$ 30-day mortality. An ordinal logistic regression analysis was done and as a dependent was used a three-step outcome (no ICU admission or mortality, ICU admission and 30-day mortality). Corticosteroid use, metastatic malignant disease or lymphoma and the severity of sepsis were identified as independent risk factors (Table 5). AUROC for ICU admission was 0.80 (CI 0.73-0.87, $p<0.001$ ) and for mortality was 0.91 (CI 0.85-0.97, $p<0.001)$. Pearson's goodness-of-fit chi-squared test was 189.86 , and degrees of freedom was 162 with significance 0.066 , showing that the model has adequate fit. Model performance was tested using Nagelkerke $R^{2}$ with a result of 0.380 .

\section{Discussion}

This retrospective single-centre cohort study of preoperative prognostic factors in adult patients with diffuse secondary peritonitis recognised several independent factors associated with composite outcome of 30-day mortality or ICU 
Table 3 Binary logistic regression analysis of preoperative risk factors for composite outcome of 30-day mortality or ICU admittance

\begin{tabular}{llc}
\hline Risk factor & Composite outcome OR $(95 \% \mathrm{CI})$ & $p$ value \\
\hline Cardiovascular disease & $4.80(2.16-10.65)$ & 0.014 \\
Chronic kidney insufficiency & $5.98(1.56-22.85)$ & 0.009 \\
Sepsis classification & Reference & $<0.001$ \\
No organ dysfunction & $4.80(2.16-10.65)$ & $<0.001$ \\
Severe sepsis & $37.94(14.52-99.13)$ & $<0.001$ \\
Septic shock & & \\
\hline
\end{tabular}

Method: forward LR, all variables categorical; contrast: indicator and reference category first $O R$ odds ratio, $C I$ confidence interval, $I C U$ intensive care unit admittance. A composite outcome of 30-day mortality or ICU admittance was chosen in order to recognise patients with severe peritonitis. If these outcomes had been analysed separately, the results would have been confusing because a group of patients were refused of ICU admission. As a consequence, the results from logistic regression analysis for ICU admittance would have represented more ICU admission and refusal criteria than actual disease severity. Independent risk factors in multivariate analysis in descending odds ratio order were septic shock, severe sepsis, chronic kidney insufficiency and cardiovascular disease. Patients lacking all of these risk factors had no mortality, and only $7.0 \%$ of these patients were admitted into the ICU.

In our data, patients can be generalised into three groups. First group, about one half of patients, had no need for ICU level care, had no comorbidities severe enough for treatment limitations and had no mortality. Second group, approximately one third of patients, had a severe peritonitis with persisting organ dysfunctions, received full level ICU care and had $16.7 \% 30$-day mortality. Third group, every tenth patient, also had a severe peritonitis but these patients were deemed too sick to benefit from intensive care and had a very high mortality ( 30 days $70.8 \%$ and 90 days $87.5 \%$ ). Indeed, more than half of the patients with a fatal outcome belonged to this group. Decision to refuse ICU admission was done case by

Table 4 Descriptive statistics of patients with immediate postoperative ICU admission refusal $(n=24)$

\begin{tabular}{ll}
\hline Characteristic & $n(\%)$ or median (IQR) \\
\hline Sex, M/F & $13 / 11(54.2 / 45.8)$ \\
Age $^{\mathrm{a}}$ & $74(64.75-87.75)$ \\
Institutionalised & $5(20.8)$ \\
Metastatic malignant disease or lymphoma & $10(41.7)$ \\
Cardiovascular disease & $12(50.0)$ \\
Charlson Comorbidity Index & $5(1-6)$ \\
Mannheim Peritonitis Index ${ }^{\mathrm{a}}$ & $32(28-35)$ \\
Sepsis classification severe/shock & $7 / 13(29.2 / 54.2)$ \\
\hline
\end{tabular}

$I Q R$ interquartile range, $M / F$ male/female

${ }^{\mathrm{a}}$ Median (IQR) case based on multiple factors, including poor functional status or end-stage severe condition combined with acute organ dysfunction.

In patients without any limitation of treatments during the first 3 days after hospitalisation, the independent risk factors for weighted outcome of ICU admission or death were septic shock, severe sepsis, metastatic malignant disease or lymphoma and corticosteroid use. Overall 30-day mortality in this subgroup was $4.7 \%$, and mortality amongst patients admitted to the ICU was $8.1 \%$.

These results show little discrepancy with previous studies or reviews [1, 2, 4, 6, 12-15]. The main difference is that this study focuses on preoperative setting, in which the important first decisions of treatment strategy are made. Also, patients with appendicitis or cholecystitis, and better prognosis, were excluded from this study, and as a consequence, overall disease severity can be considered higher. Development of organ dysfunction, especially septic shock, is a critical risk factor for mortality and ICU admittance. High suspicion and early recognition of organ dysfunctions in the emergency room are essential in order to recognise patients with impaired prognosis. These patients should be diagnosed and treated with high priority in the emergency room following timely surgical intervention and ICU admission especially if preoperative signs of organ dysfunctions do not rapidly respond to initial treatments. Interestingly, in our data, one third of patients with

Table 5 Ordinal regression analysis for patients without limitation of treatments within 3 days of hospitalisation $(n=190)$ for weighted outcome of ICU admission or 30-day mortality

\begin{tabular}{lll}
\hline Risk factor & OR $(95 \% \mathrm{CI})$ & $p$ value \\
\hline Corticosteroid use & $2.98(1.18-7.51)$ & 0.021 \\
Metastatic malignant disease or lymphoma & $3.11(1.34-7.20)$ & 0.008 \\
Sepsis classification & & \\
No organ dysfunction & Reference & \\
Severe sepsis & $5.56(2.39-12.89)$ & $<0.001$ \\
Septic shock & $11.89(4.98-28.40)$ & $<0.001$ \\
\hline
\end{tabular}

Ordinal regression dependent (no ICU or mortality, ICU admission, 90-day mortality)

$O R$ odds ratio, $C I$ confidence interval 
signs of septic shock preoperatively were not admitted to the ICU. In most of these patients, symptoms resolve during resuscitation, operation and recovery room period and some patients were refused of ICU admission. In suspected sepsis outside of the ICU, a recent study by Seymour et al. [21] suggests the use of an intriguing novel bedside clinical score termed quickSOFA (qSOFA; including respiratory rate of 22 per minute or greater, altered mentation or systolic blood pressure of $100 \mathrm{mmHg}$ or less) for screening. Such systematic evaluation might be helpful in early identification of sepsis.

Patients with cardiovascular diseases, chronic kidney insufficiency or corticosteroid drug use are at higher risk of developing a severe disease, and this risk should be recognised in surgical decision making as well as in supportive care. Other immunosuppressive medications or delay over $24 \mathrm{~h}$ from onset of symptoms until operation did not significantly correlate with outcome in univariate analysis. Due to nature of retrospective analysis, it is possible that shorter delays to surgery were associated with more severe disease, thus causing bias for effect of delay in the present study. Studies on effect of surgical delay to outcome should have a more homogenic cohort and a prospective study setting.

If patients had severe sepsis or septic shock at admission, 30-day mortality was $26.5 \%$. In a recent large Complex IntraAbdominal infection Observational (CIAO) study from Europe [22], the mortality for patients with severe sepsis or septic shock at admission was $32 \%$, which is comparable to this study.

Mortality in patients admitted to ICU was $16.7 \%$, which is remarkably low, compared to earlier studies made from the same institution. In a study by Hynninen et al., ICU-treated patients with non-postoperative and non-traumatic peritonitis had a mortality of $40 \%$ (patients treated between 2001 and 2003) [2]. Another study from our institution by Mulari et al. studied patients with secondary peritonitis requiring ICU care, and hospital mortality was $36 \%$ (patients treated between 1996 and 1998) [12]. Overall, however, there seems to be decreasing mortality rates in all ICU-treated patients with sepsis during the last decade [23]. But, it must be kept in mind that ICU admission criteria are an important issue and very closely associated with ICU mortality rates. In other words, the more patients are deemed to be too sick and refused ICU admission, the better the ICU mortality rates are. In fact, if we combine ICU-refused patients $(n=24)$ and ICU-treated patients $(n=72)$, the combined mortality rate $(17 / 24+12 / 72)$ is more comparable (30.2\%) to study by Hynninen et al. [2]. In our experience, more patients are refused ICU admission nowadays than a decade ago. In order to make reported ICU mortality rates comparable, ICU admission criteria and refusal rates should be reported as well.

In Finland, mostly ICU-specialised anaesthesiologists work as intensivists and run ICUs, serving also as gatekeepers. It is well known that ICU treatment is expensive and resources are limited [24]. Therefore, we think that it is of high importance to involve intensivists into decision-making process already in the preoperative phase. On the other hand, patients with severe comorbidities should be diagnosed and treated without delays in order that organ dysfunctions do not have time to develop.

Patients lacking severe diseases and no signs of organ dysfunctions had no mortality. So, it seems that there is a struggle between patients' physiological reservoir and the quantum of the insult given by the infection and inflammatory response. Diffuse peritonitis with severe organ dysfunctions unresponsive to initial treatments is a serious condition, but modern ICU-level treatment for organ support is highly effective and serves as an equaliser. Severe conditions with a risk of death or ICU treatment can be predicted with good accuracy already preoperatively, and these patients need to be prioritised for timely interventions.

One unanswered and very important question for future studies is that why do some patients get a very profound sepsis and some patients do not develop organ dysfunctions after diffuse peritonitis or recover from them instantly after resuscitative treatment and source control, even with similar anatomical derangement and preexisting disease burden. And, is there anything else we could do to stop the septic path? The underlying mechanisms of genomic inflammatory responses have been studied vastly, but clinical breakthroughs are yet to be discovered $[25,26]$. At the moment, the best approach is most likely to closely adherence into current sepsis guidelines [8].

The estimation of the prognosis of an individual patient is difficult, but the results from this study provide some tools to help surgeons and intensivists in decision-making process and operating room prioritising.

This study has some limitations. Most importantly, this is a retrospective and a single-centre study. The limited number of patients in the study might affect some infrequent risk factors not to become statistically significant. The retrospective nature of the study sets limitations regarding missing data points and possible inaccuracy of the collected data. At the same time, all consecutive patients were collected with no age limitations; therefore, this cohort reflects real-life patients accurately. However, only operated patients were included and we do not know how many patients were refused of operative treatment during study period.

\section{Conclusion}

In conclusion, the risk for severe peritonitis can be predicted effectively based on preoperative readily available risk factors. The most important risk factors are septic shock, signs of sepsis-related organ dysfunctions and preexisting cardiovascular disease or chronic kidney insufficiency. Patients lacking all of these risk factors had no mortality. In a subgroup of 
patients with no limitations of treatments, also, the use of corticosteroids and metastatic malignant disease were identified as independent risk factors in addition to organ dysfunctions. Since patients with diffuse peritonitis and organ dysfunctions have significant mortality, it is of paramount importance to diagnose and treat these patients effectively without delays. Patients with severe comorbidities or poor functional capacity have an extremely high mortality when refused of ICU admission.

Acknowledgments We would like to thank Juho Mattila and Tomi Eronen for providing us with the data on emergency department and ICU patients, respectively.

Authors' contributions MT had full access to the data in the study and takes responsibility for the acquisition and integrity of the data and the accuracy of the data analysis. MT drafted the manuscript with the help of VS and PM. All authors (MT, VS, PM, AL) have been involved in study conception and design, analysis and interpretation of the data and revising the manuscript. All authors have read and approved the final manuscript and agree to be accountable for all aspects of the work.

Compliance with ethical standards This study was financially supported by the Martti I. Turunen Foundation and Helsinki University Central Hospital Research Funds (Government Research Funds). The study was merely observational, and neither informed consent nor ethics committee's approval was needed. Study design was approved by institutional review board (Helsinki University Central Hospital) on June 13, 2013

Conflict of interest The authors declare that they have no conflict of interest.

\section{References}

1. Pieracci FM, Barie PS (2007) Management of severe sepsis of abdominal origin. Scand J Surg 96:184-196

2. Hynninen M, Wennervirta J, Leppäniemi A, Pettilä V (2008) Organ dysfunction and long term outcome in secondary peritonitis. Langenbecks Arch Surg 393:81-86

3. Karlsson S, Varpula M, Ruokonen E, Pettilä V, Parviainen I, AlaKokko TI, Kolho E, Rintala EM (2007) Incidence, treatment, and outcome of severe sepsis in ICU-treated adults in Finland: the Finnsepsis study. Intensive Care Med 33:435-443

4. Sartelli M, Catena F, Di Saverio S et al (2014) Current concept of abdominal sepsis: WSES position paper. World J Emerg Surg 9:22

5. Angus DC, van der Poll T (2013) Severe sepsis and septic shock. N Engl J Med 369:840-851

6. Mulier S, Penninckx F, Verwaest C, Filez L, Aerts R, Fieuws S, Lauwers P (2003) Factors affecting mortality in generalized postoperative peritonitis: multivariate analysis in 96 patients. World $\mathrm{J}$ Surg 27:379-384

7. Sartelli M, Pierluigi V, Catena F et al (2013) 2013 WSES guidelines for management of intra-abdominal infections. World J Emerg Surg $8: 3$

8. Dellinger RP, Levy MM, Rhodes A et al. (2012) Surviving sepsis campaign guidelines committee including the pediatric subgroup: surviving sepsis campaign: international guidelines for management of severe sepsis and septic shock. Critical Care Medicine 41(2013)580-637.

9. Buck DL, Vester-Andersen M, Møller MH (2013) Surgical delay is a critical determinant of survival in perforated peptic ulcer. Br J Surg 100:1045-1049

10. Azuhata T, Kinoshita K, Kawano D, Komatsu T, Sakurai A, Chiba Y, Tanjho K (2014) Time from admission to initiation of surgery for source control is a critical determinant of survival in patients with gastrointestinal perforation with associated septic shock. Crit Care 18:R87

11. Hecker A, Uhle F, Schwandner T, Padberg W, Weigand MA (2013) Diagnostics, therapy and outcome prediction in abdominal sepsis: current standards and future perspectives. Langenbecks Arch Surg 399:11-22

12. Mulari K, Leppäniemi A (2004) Severe secondary peritonitis following gastrointestinal tract perforation. Scand J Surg 93:204-208

13. Gauzit R, Péan Y, Barth X, Mistretta F, Lalaude O (2009) Top Study Team: epidemiology, management, and prognosis of secondary non-postoperative peritonitis: a French prospective observational multicenter study. Surg Infect 10:119-127

14. Wacha H, Hau T, Dittmer R, Ohmann C (1999) Risk factors associated with intraabdominal infections: a prospective multicenter study. Peritonitis Study Group. Langenbecks Arch Surg 384:24-32

15. Sallinen VJ, Leppäniemi AK, Mentula PJ (2015) Staging of acute diverticulitis based on clinical, radiologic, and physiologic parameters. J Trauma Acute Care Surg 78:543-551

16. Gutt CN, Encke J, Köninger J et al (2013) Acute cholecystitis: early versus delayed cholecystectomy, a multicenter randomized trial (ACDC study, NCT00447304). Ann Surg 258:385-393

17. Charlson ME, Pompei P, Ales KL, MacKenzie CR (1987) A new method of classifying prognostic comorbidity in longitudinal studies: development and validation. J Chronic Dis 40:373-383

18. Knaus WA, Draper EA, Wagner DP, Zimmerman JE (1985) APACHE II: a severity of disease classification system. Crit Care Med 13:818-829

19. Linder MM, Wacha H, Feldmann U, Wesch G, Streifensand RA, Gundlach E (1987) The Mannheim peritonitis index. An instrument for the intraoperative prognosis of peritonitis. Chirurg 58:84-92

20. Vincent JL, Moreno R, Takala J, Willatts S, De Mendonca A, Bruining H, Reinhart CK, Suter PM, Thijs LG (1996) The SOFA (Sepsis-related Organ Failure Assessment) score to describe organ dysfunction/failure. On behalf of the Working Group on SepsisRelated Problems of the European Society of Intensive Care Medicine. Intensive Care Med 22(199):707-710

21. Seymour CW, Liu VX, Iwashyna TJ et al (2016) Assessment of Clinical Criteria for Sepsis For the Third International Consensus Definitions for Sepsis and Septic Shock (Sepsis-3). JAMA 315: 762-774

22. Sartelli M, Catena F, Ansaloni L et al (2012) Complicated intraabdominal infections in Europe: a comprehensive review of the CIAO study. World J Emerg Surg 7:36

23. Kaukonen K-M, Bailey M, Suzuki S, Pilcher D, Bellomo R (2014) Mortality related to severe sepsis and septic shock among critically ill patients in Australia and New Zealand, 2000-2012. JAMA 311: 1308-1316

24. Sinuff T, Kahnamoui K, Cook DJ, Luce JM (2004) Values ethics and rationing in critical care task force: rationing critical care beds: a systematic review. Crit Care Med 32:1588-1597

25. Zhang Q, Raoof M, Chen Y, Sumi Y, Sursal T, Junger W, Brohi K, Itagaki K, Hauser CJ (2010) Circulating mitochondrial DAMPs cause inflammatory responses to injury. Nature 464:104-107

26. Seok J, Warren HS, Cuenca AG et al (2013) Genomic responses in mouse models poorly mimic human inflammatory diseases. Proc Natl Acad Sci U S A 110:3507-3512 